\title{
SPECTRAL MAPPING FRAMEWORK
}

\author{
ANAR DOSIEV \\ Atilim University, Incek 06836, Ankara, Turkey \\ E-mail:dosiev@yahoo.com, anardosiev@atilim.edu.tr
}

\begin{abstract}
In this paper we suggest a general framework of the spectral mapping theorem in terms of parametrized Banach space bicomplexes.
\end{abstract}

1. Introduction. In this paper we suggest a general framework of spectral mapping properties. The proposed scheme generalizes the main ideas of noncommutative spectral mapping properties developed in the Lie algebra context [7], [1], [2]. But our approach is expressed in terms of parametrized Banach space (bi)complexes without using a noncommutative operator family $a$ and (holomorphic) functions $f(a)$ of the operator family $a$. By replacing the joint spectrum $\sigma(a, f(a))$ of the operator family $(a, f(a))$ with the spectrum of a certain parametrized Banach space bicomplex connecting given parametrized Banach space complexes, we prove the forward and backward spectral mapping theorems in the general case. Note also that the proposed framework is applied in [5] to various noncommutative functional calculi (see [2], [4]) obtained for the nilpotent operator Lie subalgebras.

2. Banach space complexes. All linear spaces considered are complex. The linearly ordered set $\mathbb{Z}$ of integers suplemented with the largest (resp., lowest) element $\{\infty\}$ (resp., $\{-\infty\}$ ) is denoted by $\overline{\mathbb{Z}}$ (resp., $\underline{\mathbb{Z}}$ ), $\mathbb{N}$ is the set of all positive integers. Let $\mathcal{B}(X, Y)$ be the (semi)normed space of all bounded linear operators between (semi)normed spaces $X$ and $Y$ furnished with the operator (semi)norm and let $\mathcal{B}(X)=\mathcal{B}(X, X)$. If $T \in \mathcal{B}(X, Y)$ then $T^{*} \in \mathcal{B}\left(Y^{*}, X^{*}\right)$ denotes the dual operator. Let BS be the category of all Banach spaces and bounded morphisms. We use the notation $X \widehat{\otimes} Y$ for the projective tensor product of $X, Y \in \mathbf{B S}$ and we write $X^{\widehat{\otimes} n}$ for the $n$-fold projective tensor product of $X$. The direct sum $X \oplus Y$ is endowed with the sum-norm. One defines functors $\mathcal{B}(Y, ?), \mathcal{B}(?, Y)$

2000 Mathematics Subject Classification: Primary 47A13; Secondary 47A60, 46M18.

Key words and phrases: Słodkowski spectra, parametrized Banach space complex, ultrapowers.

The paper is in final form and no version of it will be published elsewhere. 
and ? $\widehat{\otimes} Y$ from BS to itself. Let $S$ be an infinite set and let $\mathfrak{U}$ be a nontrivial (that is, $\left.\bigcap_{M \in \mathfrak{U}} M=\emptyset\right)$ ultrafilter in $S$. The ultrafilter $\mathfrak{U}$ is said to be countably incomplete [9] if there exists a countable partition $\left\{S_{n}: n \in \mathbb{N}\right\}$ of $S$ such that $S_{n} \notin \mathfrak{U}, n \in \mathbb{N}$. In the sequel, by an ultrafilter we mean a nontrivial countably incomplete ultrafilter. The ultrapower of a Banach space $X$ (resp., Banach space operator $T$ ) with respect to an ultrafilter $\mathfrak{U}$ is denoted by $X_{\mathfrak{U}}$ (resp., $T_{\mathfrak{U}}$ ). Note that the space $X$ is embedded into $X_{\mathfrak{U}}$ as a closed subspace and $T_{\mathfrak{U}}$ extends $T$ preserving its norm, that is, $\left\|T_{\mathfrak{U}}\right\|=\|T\|$. The assignment $X \mapsto X_{\mathfrak{U}}, T \mapsto T_{\mathfrak{U}}$ defines a functor $?_{\mathfrak{U}}: \mathbf{B S} \rightarrow \mathbf{B S}$.

A chain Banach space complex is defined as a pair $(\mathfrak{X}, \mathfrak{d})$, where $\mathfrak{X}=\left\{X_{n}: n \in \mathbb{Z}\right\}$ are objects and $\mathfrak{d}=\left\{d_{n}: n \in \mathbb{Z}\right\}$ are morphisms in BS, such that $d_{n-1} d_{n}=0$ for all $n$. We also write $(\mathfrak{X}, \mathfrak{d})$ as a sequence:

$$
\cdots \longleftarrow X_{n-1} \stackrel{d_{n-1}}{\longleftarrow} X_{n} \stackrel{d_{n}}{\longleftarrow} X_{n+1} \longleftarrow \cdots .
$$

A cochain Banach space complex is defined as a sequence

$$
\cdots \rightarrow X^{n-1} \stackrel{d^{n-1}}{\rightarrow} X^{n} \stackrel{d^{n}}{\rightarrow} X^{n+1} \rightarrow \cdots
$$

of objects and morphisms of BS such that $d^{n} d^{n-1}=0$ for all $n$. The category of all (co)chain complexes in BS is denoted by $\underline{\mathbf{B S}}$ (resp., $\overline{\mathbf{B S}}$ ). The quotient (seminormed) spaces

$$
H_{n}(\mathfrak{X}, \mathfrak{d})=\operatorname{ker}\left(d_{n-1}\right) / \operatorname{im}\left(d_{n}\right)
$$

(resp., $\left.H^{n}(\mathfrak{X}, \mathfrak{d})=\operatorname{ker}\left(d^{n}\right) / \operatorname{im}\left(d^{n-1}\right)\right), n \in \mathbb{Z}$, are called the (co)homology groups of the complex $(\mathfrak{X}, \mathfrak{d})$. The complex $(\mathfrak{X}, \mathfrak{d})$ is said to be exact if all (co)homology groups are trivial. If $X_{n}=\{0\}$ (resp., $X^{n}=\{0\}$ ) for all $n, n<0$, then we say that $(\mathfrak{X}, \mathfrak{d})$ is a nonnegative complex. Note that each chain complex $(\mathfrak{X}, \mathfrak{d})$ determines a cochain complex $(\overline{\mathfrak{X}}, \overline{\mathfrak{d}})$ by setting $\bar{X}^{n}=X_{-n}$ and $\bar{d}^{n}=d_{-n}, n \in \mathbb{Z}$. Similarly, a cochain complex $(\mathfrak{X}, \mathfrak{d})$ determines a chain complex $(\underline{\mathfrak{X}}, \underline{\mathfrak{d}})$. That defines a functor $\underline{\mathbf{B S}} \longrightarrow \overline{\mathbf{B S}}$ (resp., $\overline{\mathbf{B S}} \rightarrow \underline{\mathbf{B S}}$ ) called the conjugate functor. Let $(\mathfrak{X}, \mathfrak{d}) \in \underline{\mathbf{B S}}$ and let $\left(\mathfrak{X}^{*}, \mathfrak{d}^{*}\right)$ be its dual complex:

$$
\cdots \longrightarrow X_{n-1}^{*} \stackrel{d_{n-1}^{*}}{\longrightarrow} X_{n}^{*} \stackrel{d_{n}^{*}}{\longrightarrow} X_{n+1}^{*} \longrightarrow \cdots \text {. }
$$

By definition, $\left(\mathfrak{X}^{*}, \mathfrak{d}^{*}\right)=\mathcal{B}((\mathfrak{X}, \mathfrak{d}), \mathbb{C}) \in \overline{\mathbf{B S}}$. The well known $[11,7.6 .13]$ Sequence Prime Principle asserts that $(\mathfrak{X}, \mathfrak{d})$ is exact iff so is its dual complex $\left(\mathfrak{X}^{*}, \mathfrak{d}^{*}\right)$.

2.1. Projective and flat Banach spaces. Projective (resp., flat) Banach spaces are important from the infinite-dimensional spectral mapping property viewpoint (see [1]). Let us recall the relevant definitions and simple properties of these spaces.

Let $Y \in \mathbf{B S}$ and $(\mathfrak{X}, \mathfrak{d}) \in \underline{\mathbf{B S}}$. The functor $\mathcal{B}(Y, ?): \mathbf{B S} \rightarrow \mathbf{B S}$ transforms the complex $(\mathfrak{X}, \mathfrak{d})$ into a new complex $\mathcal{B}(Y,(\mathfrak{X}, \mathfrak{d}))$ :

$$
\cdots \leftarrow \mathcal{B}\left(Y, X_{n-1}\right) \stackrel{\beta_{n-1}}{\longleftarrow} \mathcal{B}\left(Y, X_{n}\right) \stackrel{\beta_{n}}{\longleftarrow} \mathcal{B}\left(Y, X_{n+1}\right) \leftarrow \cdots,
$$

where $\beta_{n}(T)=d_{n} \cdot T, T \in \mathcal{B}\left(Y, X_{n}\right)$. By analogy, one defines a cochain Banach space complex $\mathcal{B}((\mathfrak{X}, \mathfrak{d}), Y)$. A Banach space $Y$ is said to be projective (resp., injective) if the complex $\mathcal{B}(Y,(\mathfrak{X}, \mathfrak{d}))$ (resp., $\mathcal{B}((\mathfrak{X}, \mathfrak{d}), Y)$ ) is exact for each exact Banach space complex $(\mathfrak{X}, \mathfrak{d})$. A Banach space $Y$ is said to be flat if its dual space $Y^{*}$ is injective. The class of all projective (resp., flat) Banach spaces is denoted by Proj (resp., Flat). 
Lemma 1. Let $Y \in \mathbf{B S}$. Then $Y \in$ Flat iff $(\mathfrak{X}, \mathfrak{d}) \widehat{\otimes} Y$ is exact whenever $(\mathfrak{X}, \mathfrak{d}) \in \underline{\mathbf{B S}}$ is an exact complex, where $(\mathfrak{X}, \mathfrak{d}) \widehat{\otimes} Y$ is the projective tensor product of the complex $(\mathfrak{X}, \mathfrak{d})$ and the space $Y$.

Proof. Note that $(\mathfrak{X}, \mathfrak{d}) \widehat{\otimes} Y$ is exact iff so is its dual $((\mathfrak{X}, \mathfrak{d}) \widehat{\otimes} Y)^{*}$ by the Sequence Prime Principle [11, 7.6.13]. But,

$$
((\mathfrak{X}, \mathfrak{d}) \widehat{\otimes} Y)^{*}=\mathcal{B}\left((\mathfrak{X}, \mathfrak{d}), Y^{*}\right)
$$

to within an isomorphism in $\overline{\mathbf{B S}}$. Therefore, $Y^{*}$ is injective iff $(\mathfrak{X}, \mathfrak{d}) \widehat{\otimes} Y$ is exact whenever $(\mathfrak{X}, \mathfrak{d})$ is exact.

Lemma 2. Let $Y \in \mathbf{B S}, n \in \mathbb{N}$ and let $\wedge^{n} Y$ be its $n$-th exterior power (see [3]). Then $\wedge^{n} Y \in \operatorname{Proj}$ (resp., $\wedge^{n} Y \in$ Flat) whenever $Y \in \operatorname{Proj}$ (resp., $Y \in$ Flat).

Proof. By [3], $\wedge^{n} Y \in$ Proj. Now let $Y \in$ Flat. To prove that $\wedge^{n} Y \in$ Flat, we use Lemma 1. Take an exact complex $(\mathfrak{X}, \mathfrak{d}) \in \underline{\mathbf{B S}}$. By induction on $n$, and using Lemma 1 , we infer that the complex $(\mathfrak{X}, \mathfrak{d}) \widehat{\otimes} Y^{\widehat{\otimes} n}$ remains exact. On the other hand, $\wedge^{n} Y$ is a complemented subspace in $Y^{\widehat{\otimes} n}$. Therefore $(\mathfrak{X}, \mathfrak{d}) \widehat{\otimes} \wedge^{n} Y$ is also exact.

2.2. Banach space bicomplexes. By a Banach space bicomplex we mean a triple $\left(\mathfrak{X}, \mathfrak{d} \prime, \mathfrak{d}_{\prime \prime}\right)$ with $\mathfrak{X}=\left\{X^{n, m}: n, m \in \mathbb{Z}\right\}$ being the underlying Banach spaces, $\mathfrak{d}$, $=\left\{d_{\prime}^{n, m} \in\right.$ $\left.\mathcal{B}\left(X^{n, m}, X^{n, m+1}\right)\right\}$ the column differentials, and $\mathfrak{d}{ }_{\prime \prime}=\left\{d_{\prime \prime}^{n, m} \in \mathcal{B}\left(X^{n, m}, X^{n+1, m}\right)\right\}$ the row differentials, such that the diagram

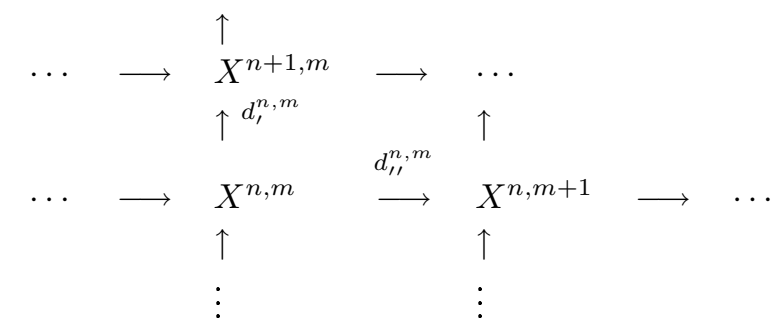

is commutative, and all columns $\left(\mathfrak{X}^{\bullet}, m, \mathfrak{d},{ }^{\bullet}, m\right)$ and all rows $\left(\mathfrak{X}^{n, \bullet}, \mathfrak{d}^{n,},{ }^{\prime}\right)$ are Banach space complexes, where $\mathfrak{X}^{\bullet}, m=\left\{X^{k, m}\right\}, \mathfrak{X}^{n, \bullet}=\left\{X^{n, k}\right\}$ and $\mathfrak{d}^{\bullet, m}=\left\{d^{k, m}\right\}, \mathfrak{d}^{n,}{ }^{n, \bullet}=\left\{d_{\prime \prime}^{n, k}\right\}$. If we reverse the horizontal and (or) vertical arrows we obtain other versions of bicomplexes as well as chain and cochain complexes. For us, of interest will be the "double-cochain" and "double-chain" versions of a bicomplex. This is caused by our future applications [5] to spectral theory. We also say chain (resp., cochain) bicomplex instead of "doublechain" (resp., "double-cochain"). One easily checks that $d_{\prime}^{n, m}\left(\operatorname{ker}\left(d_{\prime \prime}^{n, m}\right)\right) \subseteq \operatorname{ker}\left(d_{\prime \prime}^{n+1, m}\right)$ and $d_{\prime}^{n, m}\left(\operatorname{im}\left(d_{\prime \prime}^{n, m-1}\right)\right) \subseteq \operatorname{im}\left(d_{\prime \prime}^{n+1, m-1}\right)$, therefore the quotient operator

$$
D_{1}^{n, m}: H^{m}\left(\mathfrak{X}^{n, \bullet}, \mathfrak{d}_{\prime \prime}^{n, \bullet}\right) \rightarrow H^{m}\left(\mathfrak{X}^{n+1, \bullet}, \mathfrak{d}_{\prime \prime}^{n+1, \bullet}\right), \quad D_{l}^{n, m}\left(x^{\sim}\right)=d_{l}^{n, m}(x)^{\sim},
$$

is well defined. Moreover, the sequence

$$
\cdots \longrightarrow H^{m}\left(\mathfrak{X}^{n, \bullet}, \mathfrak{d}_{\prime \prime}^{n, \bullet}\right) \stackrel{D_{\prime}^{n, m}}{\longrightarrow} H^{m}\left(\mathfrak{X}^{n+1, \bullet}, \mathfrak{d}_{\prime \prime}^{n+1, \bullet}\right) \longrightarrow \cdots
$$


is a complex called the $m$-th vertical cohomology complex of the bicomplex. By analogy, one defines the $n$-th horizontal cohomology complex

$$
\cdots \longrightarrow H^{n}\left(\mathfrak{X}^{\bullet, m}, \mathfrak{d}^{\bullet}, m\right) \stackrel{D_{\prime \prime}^{n, m}}{\longrightarrow} H^{n}\left(\mathfrak{X}^{\bullet}, m+1, \mathfrak{d}^{\bullet}, m+1\right) \longrightarrow \cdots
$$

of the bicomplex. We say that a bicomplex $\left(\mathfrak{X}, \mathfrak{d}_{\prime}, \mathfrak{d}_{\prime \prime}\right)$ is bounded below if one can find $N \in \mathbb{Z}$ such that $X^{n, m}=\{0\}$ whenever $n<N$ or $m<N$. The space $X^{N, N}$ is called the initial space of the bicomplex. If $N=0$ then we say that $\left(\mathfrak{X}, \mathfrak{d} \prime, \mathfrak{d} \prime^{\prime \prime}\right)$ is a nonnegative bicomplex with the initial space $X^{0,0}$.

Let $\left(\mathfrak{X}, \mathfrak{d}_{\prime}, \mathfrak{d}_{\prime \prime}\right)$ be a Banach space bicomplex bounded below, $X^{n}=\bigoplus_{k+s=n} X^{k, s} \in$ BS a sum of (bounded) diagonals of the bicomplex. One defines a Banach space complex

$$
\cdots \longrightarrow X^{n} \stackrel{\delta^{n}}{\longrightarrow} X^{n+1} \longrightarrow \cdots
$$

where $\delta^{n}(x)=d_{\prime \prime}^{k, s}(x)+(-1)^{s} d_{\prime}^{k, s}(x)$ whenever $x \in X^{k, s}, k+s=n, n \in \mathbb{Z}$. The latter is called the total complex of $\left(\mathfrak{X}, \mathfrak{d},^{\prime}, \mathfrak{d}_{\prime \prime}^{\prime \prime}\right)$ and it is $\operatorname{denoted}$ by $\operatorname{Tot}\left(\mathfrak{X}, \mathfrak{d}_{\prime}, \mathfrak{d}_{\prime \prime}\right)$. If $\left(\mathfrak{X}, \mathfrak{d} \mathfrak{d}^{\prime}, \mathfrak{d}_{\prime \prime}\right)$ is a Banach space bicomplex and $\mathfrak{U}$ is an ultrafilter then $\left(\mathfrak{X}_{\mathfrak{U}}, \mathfrak{d} \prime_{\mathfrak{U}}, \mathfrak{d} \prime^{\prime} \mathfrak{U}\right)$ is a bicomplex called an ultrapower of $\left(\mathfrak{X}, \mathfrak{d}{ }^{\prime}, \mathfrak{d} \prime^{\prime \prime}\right)$, where $\mathfrak{X}_{\mathfrak{U}}=\left\{X_{\mathfrak{U}}^{n, m}\right\}, \mathfrak{d}^{\prime} \mathfrak{U}=\left\{d_{\prime \mathfrak{U}}^{n, m}\right\}, \mathfrak{d}^{\prime \prime} \mathfrak{U}=\left\{d_{\prime \prime}^{n, m}\right\}$. Bearing in mind that the ultrapower functor preserves the finite direct sums (in BS) [9], we conclude that

$$
\operatorname{Tot}\left(\left(\mathfrak{X}_{\mathfrak{U}}, \mathfrak{d}_{\prime \mathfrak{U}}, \mathfrak{d}_{\prime \prime} \mathfrak{U}\right)\right)=\operatorname{Tot}\left(\mathfrak{X}, \mathfrak{d}, \mathfrak{d}_{\prime \prime}\right)_{\mathfrak{U}}
$$

The following lemmas can be proved by using the known diagonal chase method (see [6, Appendix 2], [10, Ch. 2, Section 5.4]).

LEMMA 3. Let $\left(\mathfrak{X}, \mathfrak{d},^{\prime} \mathfrak{d}_{\prime \prime}\right)$ be a nonnegative cochain bicomplex such that all its rows are exact at first $i-1$ terms, $H^{i}\left(\mathfrak{X}^{0, \bullet}, \mathfrak{d}^{0, \bullet}\right) \neq\{0\}$ and the differential

$$
D_{,}^{0, i}: H^{i}\left(\mathfrak{X}^{0, \bullet}, \mathfrak{d}_{\prime \prime}^{0, \bullet}\right) \rightarrow H^{i}\left(\mathfrak{X}^{1, \bullet}, \mathfrak{d}_{\prime \prime}^{1, \bullet}\right)
$$

of the $i$-th vertical cohomology complex is trivial, where $i \in \mathbb{N}$. Then $H^{k}\left(\mathfrak{X}^{\bullet, m}, \mathfrak{d}^{\bullet, m}\right) \neq\{0\}$ for some $k, m \leq i$.

REMARK 1. A chain version of the assertion of Lemma 3 is the following. Let $\left(\mathfrak{X}, \mathfrak{d}^{\prime}, \mathfrak{d}^{\prime \prime}\right)$ be a nonnegative chain bicomplex such that all its rows are exact at first $i-1$ terms, $H_{i}\left(\mathfrak{X}_{0, \bullet}, \mathfrak{d}_{0, \bullet}^{\prime \prime}\right) \neq\{0\}$ and the differential $D_{0, i}^{\prime}: H_{i}\left(\mathfrak{X}_{1, \bullet}, \mathfrak{d}_{1, \bullet}^{\prime \prime}\right) \rightarrow H_{i}\left(\mathfrak{X}_{0, \bullet}, \mathfrak{d}_{0, \bullet}^{\prime \prime}\right)$ of the $i$-th vertical homology complex is trivial. Then $H_{k}\left(\mathfrak{X}_{\bullet}, m, \mathfrak{d}_{\bullet}^{\prime}, m\right) \neq\{0\}$ for some $k, m \leq i$.

LEMma 4. Let $\left(\mathfrak{X}, \mathfrak{d}^{\prime}, \mathfrak{d}_{\prime \prime}\right)$ be a nonnegative (co)chain bicomplex such that the first $n$ vertical or horizontal (co)homology complexes are exact. Then $\operatorname{Tot}\left(\mathfrak{X}, \mathfrak{d} \prime, \mathfrak{d} \prime^{\prime \prime}\right)$ is exact at the first $n$ terms.

3. Stability of spectra under some functors. In this section we introduce Słodkowski spectra of parametrized Banach space complexes and prove their stability under functors considered above.

Let $\Omega$ be a topological space and let $\mathfrak{X}=\left\{X_{n}: n \in \mathbb{Z}\right\}$ be a collection of Banach spaces. Assume that there exists a collection of continuous maps $\mathfrak{d}=\left\{d_{n}: n \in \mathbb{Z}\right\}$, 
$d_{n}: \Omega \rightarrow \mathcal{B}\left(X_{n+1}, X_{n}\right)$, such that $(\mathfrak{X}, \mathfrak{o}(\lambda))$ is a chain Banach space complex

$$
\cdots \longleftarrow X_{n-1} \stackrel{d_{n-1}(\lambda)}{\longleftarrow} X_{n} \stackrel{d_{n}(\lambda)}{\longleftarrow} X_{n+1} \longleftarrow \cdots
$$

for each $\lambda \in \Omega$, where $\mathfrak{d}(\lambda)=\left\{d_{n}(\lambda)\right\}$. The collection of Banach space complexes $(\mathfrak{X}, \mathfrak{d}(\lambda)), \lambda \in \Omega$, is called a parametrized chain Banach space complex or chain $\Omega$-Banach complex and it is denoted by $(\mathfrak{X}, \mathfrak{d})$. If $(\mathfrak{X}, \mathfrak{d}(\lambda))$ is a cochain complex for each $\lambda \in \Omega$, then $(\mathfrak{X}, \mathfrak{d})$ is said to be a cochain $\Omega$-Banach complex. Morphisms of $\Omega$-Banach complexes are defined in a self-evident way. Using the functors $\mathcal{B}(Y, ?), \mathcal{B}(?, Y), ? \widehat{\otimes} Y$, and ? $\mathfrak{U}$, we could associate new $\Omega$-Banach complexes to the original $\Omega$-Banach complex $(\mathfrak{X}, \mathfrak{d})$. In particular,

$$
\mathcal{B}((\mathfrak{X}, \mathfrak{d}), \mathbb{C})=\left(\mathfrak{X}^{*}, \mathfrak{d}^{*}\right)=\left\{\left(\mathfrak{X}^{*}, \mathfrak{d}(\lambda)^{*}\right): \lambda \in \Omega\right\}
$$

is the dual parametrized complex.

A parametrized (co)chain Banach space bicomplex is defined as a certain bicomplex $\left(\mathfrak{X}, \mathfrak{d}^{\prime}, \mathfrak{d}^{\prime \prime}\right)$ such that all its rows $\left(\mathfrak{X}_{n, \bullet}, \mathfrak{d}_{n, \bullet}^{\prime \prime}\right)$ are $\Omega$-Banach complexes, columns $\left(\mathfrak{X}_{\bullet}, m, \mathfrak{d}_{\bullet}^{\prime}, m\right)$ are $\Lambda$-Banach complexes, and $\left(\mathfrak{X}, \mathfrak{d}^{\prime}(\lambda), \mathfrak{d}^{\prime \prime}(\mu)\right)$ is a Banach space bicomplex for all $\lambda \in \Omega$ and $\mu \in \Lambda$. In this case we say that $\left(\mathfrak{X}, \mathfrak{d}^{\prime}, \mathfrak{d}^{\prime \prime}\right)$ is an $\Omega \times \Lambda$-Banach bicomplex.

Now let $(\mathfrak{X}, \mathfrak{d})$ be a $(\mathrm{co})$ chain parametrized Banach space complex,

$$
\Sigma_{n}(\mathfrak{X}, \mathfrak{d})=\left\{\lambda \in \Omega: H_{n}(\mathfrak{X}, \mathfrak{d}(\lambda)) \neq\{0\}\right\},
$$

and $\Sigma^{n}(\mathfrak{X}, \mathfrak{d})=\left\{\lambda \in \Omega: H^{n}(\mathfrak{X}, \mathfrak{d}(\lambda)) \neq\{0\}\right\}$ if $(\mathfrak{X}, \mathfrak{d})$ is a cochain complex, $n \in \mathbb{Z}$. Further, let

$$
\sigma_{\delta, n}(\mathfrak{X}, \mathfrak{d})=\bigcup_{k \leq n} \Sigma_{k}(\mathfrak{X}, \mathfrak{d})
$$

and let $\sigma_{\pi, n}(\mathfrak{X}, \mathfrak{d})$ be the set of those $\lambda \in \Omega$ such that $\lambda \in \bigcup_{k \geq n} \Sigma_{k}(\mathfrak{X}, \mathfrak{d})$ or the image of the operator $d_{n-1}(\lambda)$ is not closed. By analogy, we set

$$
\sigma^{\delta, n}(\mathfrak{X}, \mathfrak{d})=\bigcup_{k \geq n} \Sigma^{k}(\mathfrak{X}, \mathfrak{d})
$$

and $\sigma^{\pi, n}(\mathfrak{X}, \mathfrak{d})$ is the set of those $\lambda \in \Omega$ such that $\lambda \in \bigcup_{k \leq n} \Sigma^{k}(\mathfrak{X}, \mathfrak{d})$ or the image of the operator $d^{n}(\lambda)$ is not closed, whenever $(\mathfrak{X}, \mathfrak{d})$ is a cochain complex. One easily checks that $\sigma^{\delta, n}(\mathfrak{X}, \mathfrak{d})=\sigma_{\delta, n}(\underline{\mathfrak{X}}, \underline{\mathfrak{d}})$ and $\sigma_{\pi, n}(\mathfrak{X}, \mathfrak{d})=\sigma^{\pi, n}(\overline{\mathfrak{X}}, \overline{\mathfrak{d}}), n \in \mathbb{Z}$.

Definition 1. The set-valued functions $\sigma_{\delta, n}, \sigma_{\pi, n}$ (resp., $\left.\sigma^{\delta, n}, \sigma^{\pi, n}\right), n \in \mathbb{Z}$, defined on the class of all parametrized (co)chain Banach space complexes are called the Stodkowski spectra. The set

$$
\sigma_{\mathrm{t}}(\mathfrak{X}, \mathfrak{d})=\sigma_{\delta, \infty}(\mathfrak{X}, \mathfrak{d})=\sigma_{\pi,-\infty}(\mathfrak{X}, \mathfrak{d})=\bigcup_{n \in \mathbb{Z}} \Sigma_{n}(\mathfrak{X}, \mathfrak{d})
$$

(resp., $\sigma_{\mathrm{t}}(\mathfrak{X}, \mathfrak{d})=\sigma^{\pi, \infty}(\mathfrak{X}, \mathfrak{d})=\sigma^{\delta,-\infty}(\mathfrak{X}, \mathfrak{d})=\bigcup_{n \in \mathbb{Z}} \Sigma^{n}(\mathfrak{X}, \mathfrak{d})$ for the cochain complex $(\mathfrak{X}, \mathfrak{d}))$ is called the Taylor spectrum of $(\mathfrak{X}, \mathfrak{d})$. We set $\mathfrak{S}=\mathfrak{S}_{\delta} \cup \mathfrak{S}_{\pi}$ (resp., $\left.\mathfrak{S}=\mathfrak{S}^{\delta} \cup \mathfrak{S}^{\pi}\right)$, where $\mathfrak{S}_{\delta}=\left\{\sigma_{\delta, n}: n \in \overline{\mathbb{Z}}\right\}, \mathfrak{S}_{\pi}=\left\{\sigma_{\pi, n}: n \in \underline{\mathbb{Z}}\right\}$ (resp., $\mathfrak{S}^{\delta}=\left\{\sigma^{\delta, n}: n \in \underline{\mathbb{Z}}\right\}$, $\left.\mathfrak{S}^{\pi}=\left\{\sigma^{\pi, n}: n \in \overline{\mathbb{Z}}\right\}\right)$.

In the sequel, $\sigma \in \mathfrak{S}$ denotes one of Słodkowski spectra if the latter is not specially indicated. Let $(\mathfrak{X}, \mathfrak{d})$ be an $\Omega$-Banach complex. Using the Sequence Prime Principle, we 
obtain (for details, see [12]) that

$$
\sigma^{\pi, n}\left(\mathfrak{X}^{*}, \mathfrak{d}^{*}\right)=\sigma_{\delta, n}(\mathfrak{X}, \mathfrak{d}), \quad \sigma_{\delta, n}\left(\mathfrak{X}^{*}, \mathfrak{d}^{*}\right)=\sigma^{\pi, n}(\mathfrak{X}, \mathfrak{d}) .
$$

To calculate all other Słodkowski spectra of the dual parametrized Banach space complex it suffices to use the conjugate functor. For instance, if $(\mathfrak{X}, \mathfrak{d})$ is a chain $\Omega$-Banach complex then

$$
\sigma^{\delta, n}\left(\mathfrak{X}^{*}, \mathfrak{d}^{*}\right)=\sigma_{\delta, n}\left(\overline{\mathfrak{X}^{*}}, \overline{\mathfrak{d}^{*}}\right)=\sigma_{\delta, n}\left(\overline{\mathfrak{X}}^{*}, \overline{\mathfrak{d}}^{*}\right)=\sigma^{\pi, n}(\overline{\mathfrak{X}}, \overline{\mathfrak{d}})=\sigma_{\pi, n}(\mathfrak{X}, \mathfrak{d}) .
$$

By analogy, we conclude that $\sigma_{\pi, n}\left(\mathfrak{X}^{*}, \mathfrak{d}^{*}\right)=\sigma^{\delta, n}(\mathfrak{X}, \mathfrak{d})$ whenever $(\mathfrak{X}, \mathfrak{d})$ is a cochain complex. The following assertion slightly extends relevant results from [2], [3] and can be proved in the same way.

Theorem 1. Let $(\mathfrak{X}, \mathfrak{d})$ be a (co)chain $\Omega$-Banach complex and let $Y \in \mathbf{B S}$.

a) Then $\sigma(\mathfrak{X}, \mathfrak{d}) \subseteq \sigma(\mathcal{B}(Y,(\mathfrak{X}, \mathfrak{d})))$ and $\sigma(\mathfrak{X}, \mathfrak{d}) \subseteq \sigma((\mathfrak{X}, \mathfrak{d}) \widehat{\otimes} Y)$ for all $\sigma \in \mathfrak{S}$. Moreover, these inclusions become equalities whenever $Y \in$ Proj and $Y \in$ Flat, respectively.

b) If $\mathfrak{U}$ is an ultrafilter then

$$
\sigma_{\pi, n}\left(\mathfrak{X}_{\mathfrak{U}}, \mathfrak{d}_{\mathfrak{U}}\right)=\bigcup_{k \geq n} \Sigma_{k}\left(\mathfrak{X}_{\mathfrak{U}}, \mathfrak{d}_{\mathfrak{U}}\right)
$$

for all $\sigma_{\pi, n} \in \mathfrak{S}_{\pi}$. Moreover,

$$
\sigma(\mathfrak{X}, \mathfrak{d})=\sigma\left(\mathfrak{X}_{\mathfrak{U}}, \mathfrak{d}_{\mathfrak{U}}\right)
$$

for all $\sigma \in \mathfrak{S}$.

4. The spectral mapping properties. In this section we present a cochain version of the spectral mapping properties for $\pi$-type Słodkowski spectra (see Definition 1). Our approach strongly depends on the ultrapower functor. We have noted above (see (3.1)) that $\delta$-type Słodkowski spectra of chain complexes reduce to $\pi$-type spectra of its dual complex. That would allow us to formulate relevant assertions for $\delta$-type spectra. But the ultrapower functor and the functor of taking the dual space are not compatible (see $[9])$, therefore $\delta$-type spectra will appear in applications [5].

Let $(\mathfrak{X}, \mathfrak{d})$ and $(\mathfrak{Y}, \overline{\mathfrak{d}})$ be a nonnegative cochain parametrized Banach space complexes such that both complexes have the same first term $X=X^{0}=Y^{0}$ and let $\Omega$ and $\Lambda$ be their spaces of parameters, respectively. We say that these complexes are $\pi$-spectrally connected if there exists a nonnegative cochain $\Omega \times \Lambda$-Banach bicomplex $\left(\mathfrak{Z}, \mathfrak{d}^{\prime}, \mathfrak{d}^{\prime \prime}\right)$ such $\left(\mathfrak{Z}^{0, \bullet}, \mathfrak{d}_{\prime \prime}^{0, \bullet}\right)=(\mathfrak{X}, \mathfrak{d}),\left(\mathfrak{Z}^{\bullet, 0}, \mathfrak{d}^{\bullet}, 0\right)=(\mathfrak{Y}, \overline{\mathfrak{d}})$ and

$$
\sigma\left(\mathfrak{Z}^{s, \bullet}, \mathfrak{d}_{\prime \prime}^{s, \bullet}\right) \subseteq \sigma(\mathfrak{X}, \mathfrak{d}), \quad \sigma\left(\mathfrak{Z}^{\bullet}, m, \mathfrak{d},{ }^{\bullet}, m\right) \subseteq \sigma(\mathfrak{Y}, \overline{\mathfrak{d}})
$$

for all $\sigma \in \mathfrak{S}^{\pi}$, and $s, m \in \mathbb{N}$. Thus $(\mathfrak{Z}, \mathfrak{d} \prime(\lambda), \mathfrak{d} \prime(\mu))$ is a nonnegative Banach space bicomplex with the initial space $X$ for each $(\lambda, \mu) \in \Omega \times \Lambda$. Their total complexes $\operatorname{Tot}\left(\mathfrak{Z}, \mathfrak{d} \prime(\lambda), \mathfrak{d} \prime^{\prime \prime}(\mu)\right),(\lambda, \mu) \in \Omega \times \Lambda$, define an $\Omega \times \Lambda$-Banach complex $\operatorname{Tot}\left(\mathfrak{Z}, \mathfrak{d}_{\prime}, \mathfrak{d}_{\prime \prime}\right)$; let $\sigma\left(\mathfrak{Z}, \mathfrak{d} \prime, \mathfrak{d}_{\prime \prime}\right)$ denote the Słodkowski spectrum of the latter complex.

Proposition 1. Let $(\mathfrak{X}, \mathfrak{d})$ and $(\mathfrak{Y}, \overline{\mathfrak{d}})$ be a $\pi$-spectrally connected cochain complexes and let $\sigma \in \mathfrak{S}^{\pi}$. Then

$$
\sigma\left(\mathfrak{Z}, \mathfrak{d} \prime, \mathfrak{d}^{\prime \prime}\right) \subseteq \sigma(\mathfrak{X}, \mathfrak{d}) \times \sigma(\mathfrak{Y}, \overline{\mathfrak{d}})
$$

for a parametrized Banach space bicomplex $(\mathfrak{Z}, \mathfrak{d} \prime, \mathfrak{d} \prime \prime)$ connecting $(\mathfrak{X}, \mathfrak{d})$ and $(\mathfrak{Y}, \overline{\mathfrak{d}})$. 
Proof. Let $\mathfrak{U}$ be an ultrafilter and let $\sigma=\sigma^{\pi, n}$. Undoubtedly, the ultrapower $(\mathfrak{Z}, \mathfrak{d} \prime, \mathfrak{d} \prime \prime) \mathfrak{U}$ $(=(\mathfrak{Z} \mathfrak{U}, \mathfrak{d} / \mathfrak{U}, \mathfrak{d} / \mathfrak{U}))$ is a parametrized Banach space bicomplex connecting complexes $\left(\mathfrak{X}_{\mathfrak{U}}, \mathfrak{d}_{\mathfrak{U}}\right)$ and $\left(\mathfrak{Y}_{\mathfrak{U}}, \overline{\mathfrak{d}}_{\mathfrak{U}}\right)$. Moreover, $\left(\mathfrak{X}_{\mathfrak{U}}, \mathfrak{d}_{\mathfrak{U}}\right)$ and $\left(\mathfrak{Y}_{\mathfrak{U}}, \overline{\mathfrak{d}}_{\mathfrak{U}}\right)$ are $\pi$-spectrally connected by means of $\left(\mathfrak{Z}, \mathfrak{d},^{\prime} \mathfrak{d}_{\prime \prime}\right)_{\mathfrak{U}}$ due to Theorem 1 (b). Appealing to (2.1) and Theorem 1 (b), we also infer that

$$
\begin{aligned}
& \sigma\left(\mathfrak{Z}, \mathfrak{d}_{\prime}, \mathfrak{d}_{\prime \prime}\right)=\sigma\left(\operatorname{Tot}\left(\mathfrak{Z}, \mathfrak{d}_{\prime}, \mathfrak{d}_{\prime \prime}\right)\right)=\sigma\left(\operatorname{Tot}\left(\mathfrak{Z}, \mathfrak{d}_{\prime}, \mathfrak{d}_{\prime \prime}\right)_{\mathfrak{U}}\right)=\sigma\left(\operatorname{Tot}\left(\mathfrak{Z}_{\mathfrak{U}}, \mathfrak{d}_{\prime}{ }_{\mathfrak{U}}, \mathfrak{d}_{\prime \prime}{ }^{\prime}\right)\right) \\
& =\sigma\left(\mathfrak{Z} \mathfrak{U}, \mathfrak{d} / \mathfrak{U}, \mathfrak{d} \prime^{\prime} \mathfrak{U}\right)
\end{aligned}
$$

and

$$
\sigma\left(\mathfrak{Z}_{\mathfrak{U}}, \mathfrak{d}_{\mathfrak{U}^{\mathfrak{U}}}, \mathfrak{d}_{\prime \prime} \mathfrak{U}\right)=\bigcup_{k \leq n} \Sigma^{k}\left(\operatorname{Tot}\left(\mathfrak{Z}_{\mathfrak{U}}, \mathfrak{d}^{\prime \mathfrak{U}}, \mathfrak{d}^{\prime \prime} \mathfrak{U}\right)\right) .
$$

Now take $(\lambda, \mu) \in \sigma\left(\mathfrak{Z}, \mathfrak{d},^{\prime} \mathfrak{d}{ }^{\prime}\right)$. If $\lambda \notin \sigma(\mathfrak{X}, \mathfrak{d})$ then $\lambda \notin \bigcup_{k \leq n} \Sigma^{k}\left(\mathfrak{X}_{\mathfrak{U}}, \mathfrak{d}_{\mathfrak{U}}\right)$ by Theorem 1 (b). It follows that

$$
\lambda \notin \sigma\left(\mathfrak{Z}_{\mathfrak{U}}^{m, \bullet}, \mathfrak{d}^{\prime \prime}, \mathfrak{U}\right)=\bigcup_{k \leq n} \Sigma^{k}\left(\mathfrak{Z}_{\mathfrak{U}}^{m, \bullet}, \mathfrak{d}^{\prime \prime}, \mathfrak{U}, \bullet\right)
$$

for all $m \in \mathbb{Z}_{+}$. Thus all rows of the bicomplex $\left(\mathfrak{Z}_{\mathfrak{U}}, \mathfrak{d},(\lambda)_{\mathfrak{U}}, \mathfrak{d}{ }^{\prime \prime}(\mu)_{\mathfrak{U}}\right)$ are exact at the first $n$ terms, which implies that $\operatorname{Tot}\left(\mathfrak{Z}_{\mathfrak{U}}, \mathfrak{d} \prime(\lambda)_{\mathfrak{U}}, \mathfrak{d}{ }_{\prime \prime}(\mu)_{\mathfrak{U}}\right)$ is exact at first $n$ terms by virtue of Lemma 4. The latter means that $(\lambda, \mu) \notin \bigcup_{k \leq n} \Sigma^{k}\left(\operatorname{Tot}\left(\mathfrak{Z}_{\mathfrak{U}}, \mathfrak{d}_{\prime} \mathfrak{U}, \mathfrak{d}_{\prime \prime} \mathfrak{U}\right)\right)$, or $(\lambda, \mu) \notin$ $\sigma\left(\mathfrak{Z}, \mathfrak{d}_{\prime}, \mathfrak{d}_{\prime \prime}\right)$, a contradiction. The same argument with columns of the bicomplex yields $\mu \in \sigma(\mathfrak{Y}, \overline{\mathfrak{d}})$.

REMARK 2. One can similarly prove a chain version of the assertion from Proposition 1. Namely, let $(\mathfrak{X}, \mathfrak{d})$ and $(\mathfrak{Y}, \overline{\mathfrak{d}})$ be $\delta$-spectrally connected chain complexes. Then

$$
\sigma\left(\mathfrak{Z}, \mathfrak{d} \prime, \mathfrak{d} \prime^{\prime \prime}\right) \subseteq \sigma(\mathfrak{X}, \mathfrak{d}) \times \sigma(\mathfrak{Y}, \overline{\mathfrak{d}})
$$

for a parametrized bicomplex $\left(\mathfrak{Z}, \mathfrak{d} \prime, \mathfrak{d}_{\prime \prime}\right)$ connecting $(\mathfrak{X}, \mathfrak{d})$ and $(\mathfrak{Y}, \overline{\mathfrak{d}}), \sigma \in \mathfrak{S}_{\delta}$.

DeFinition 2. Let $(\mathfrak{X}, \mathfrak{d})$ and $(\mathfrak{Y}, \overline{\mathfrak{d}})$ be $\pi$-spectrally connected complexes parametrized on a topological spaces $\Omega$ and $\Lambda$, respectively, and let $\left(\mathfrak{Z}, \mathfrak{d}_{\prime}, \mathfrak{d}_{\prime \prime}\right)$ be an $\Omega \times \Lambda$-bicomplex connecting these complexes. By a spectral mapping with respect to $\left(\mathfrak{Z}, \mathfrak{d}_{\prime}, \mathfrak{d}_{\prime \prime}\right)$ we mean a continuous map $f: \Omega \rightarrow \Lambda$ such that

a) all vertical cohomology complexes

$$
0 \rightarrow H^{m}(\mathfrak{X}, \mathfrak{d}(\lambda)) \rightarrow \cdots \rightarrow H^{m}\left(\mathfrak{Z}^{n, \bullet}, \mathfrak{d}_{\prime \prime}^{n, \bullet}(\lambda)\right) \stackrel{D_{\prime}^{n, m}(\mu)}{\longrightarrow} H^{m}\left(\mathfrak{Z}^{n+1, \bullet}, \mathfrak{d}_{\prime \prime}^{n+1, \bullet}(\lambda)\right) \rightarrow \cdots
$$

of the bicomplex $(\mathfrak{Z}, \mathfrak{d} \prime(\lambda), \mathfrak{d} \prime \prime(\mu))$ are exact whenever $\mu \neq f(\lambda)$;

b) $D_{,}^{0, m}(f(\lambda))=0$ whenever the cohomology space $H^{m}(\mathfrak{X}, \mathfrak{d}(\lambda))$ is Hausdorff.

If only b) is satisfied then we say that $f$ is a prespectral mapping.

Note that condition a) of Definition 2 means that all terms ' $E_{2}^{m n}(\lambda, \mu), m, n \in \mathbb{Z}_{+}$, of the first spectral sequence associated with the bicomplex $(\mathfrak{Z}, \mathfrak{d} \prime(\lambda), \mathfrak{d} \prime \prime(\mu))$ (see $[8$, Ch. $1,4.8])$ are vanishing whenever $\mu \neq f(\lambda)$.

Now let us prove the forward and backward spectral mapping properties of spectrally connected complexes. 
THEOREM 2. Let $(\mathfrak{X}, \mathfrak{d})$ and $(\mathfrak{Y}, \overline{\mathfrak{d}})$ be cochain complexes parametrized on $\Omega$ and $\Lambda$, respectively, and let $\mathfrak{U}$ be an ultrafilter. If $\left(\mathfrak{X}_{\mathfrak{U}}, \mathfrak{d}_{\mathfrak{U}}\right)$ and $(\mathfrak{Y}, \overline{\mathfrak{d}})$ are $\pi$-spectrally connected and $f: \Omega \rightarrow \Lambda$ is a prespectral mapping then

$$
f(\sigma(\mathfrak{X}, \mathfrak{d})) \subseteq \sigma(\mathfrak{Y}, \overline{\mathfrak{d}})
$$

for all $\sigma \in \mathfrak{S}^{\pi}$.

Proof. Let $\left(\mathfrak{Z}, \mathfrak{d},^{\prime}, \mathfrak{d}_{\prime \prime}\right)$ be an $\Omega \times \Lambda$-bicomplex connecting $\left(\mathfrak{X}_{\mathfrak{U}}, \mathfrak{d}_{\mathfrak{U}}\right)$ and $(\mathfrak{Y}, \overline{\mathfrak{d}})$, and let $\sigma=\sigma^{\pi, n}$. Take $\lambda \in \sigma(\mathfrak{X}, \mathfrak{d})$ and let $\mu=f(\lambda)$. By Theorem $1(\mathrm{~b})$,

$$
\sigma(\mathfrak{X}, \mathfrak{d})=\sigma\left(\mathfrak{X}_{\mathfrak{U}}, \mathfrak{d}_{\mathfrak{U}}\right)=\bigcup_{k \leq n} \Sigma^{k}\left(\mathfrak{X}_{\mathfrak{U}}, \mathfrak{d}_{\mathfrak{U}}\right) .
$$

Choose lowest $i$ such that $\lambda \in \Sigma^{i}\left(\mathfrak{X}_{\mathfrak{U}}, \mathfrak{d}_{\mathfrak{U}}\right)$. Then $H^{i}\left(\mathfrak{X}_{\mathfrak{U}}, \mathfrak{d}_{\mathfrak{U}}(\lambda)\right)$ is a nontrivial Banach space and $\lambda \notin \sigma^{\pi, i-1}\left(\mathfrak{X}_{\mathfrak{U}}, \mathfrak{d}_{\mathfrak{U}}\right)$, otherwise $\lambda \in \bigcup_{k \leq i-1} \Sigma^{k}\left(\mathfrak{X}_{\mathfrak{U}}, \mathfrak{d}_{\mathfrak{U}}\right)$ by virtue of Theorem 1 (b). Since $\left(\mathfrak{X}_{\mathfrak{U}}, \mathfrak{d}_{\mathfrak{U}}\right)$ and $(\mathfrak{Y}, \overline{\mathfrak{d}})$ are $\pi$-spectrally connected (by means of $\left.\left(\mathfrak{Z}, \mathfrak{d}_{\prime}, \mathfrak{d}_{\prime \prime}^{\prime \prime}\right)\right)$ complexes, it follows that $\sigma^{\pi, i-1}\left(\mathfrak{Z}^{n, \bullet}, \mathfrak{d}^{n,}, \bullet\right) \subseteq \sigma^{\pi, i-1}\left(\mathfrak{X}_{\mathfrak{U}}, \mathfrak{d}_{\mathfrak{U}}\right)$ for all $n$. Thus all rows of $\left(\mathfrak{Z}, \mathfrak{d} \prime, \mathfrak{d}_{\prime \prime}\right)$ are exact at the first $i-1$ terms. Moreover, the differential

$$
D_{,}^{0, i}(\mu): H^{i}\left(\mathfrak{X}_{\mathfrak{U}}, \mathfrak{d}_{\mathfrak{U}}(\lambda)\right) \rightarrow H^{i}\left(\mathfrak{Z}^{1, \bullet}, \mathfrak{d}_{\prime \prime}^{1, \bullet}\right)
$$

of the $i$-th vertical cohomology complex is trivial by Definition 2 . Then $H^{k}\left(\mathfrak{Z}^{\bullet, m}, \mathfrak{d}{ }^{\bullet}, m\right) \neq$ $\{0\}$ for some $k, m \leq i$ by virtue of Lemma 3. The latter means that $\mu \in \sigma^{\pi, i}\left(\mathfrak{Z}^{\bullet, m}, \mathfrak{d}{ }^{\bullet}, m\right) \subseteq$ $\sigma\left(\mathfrak{Z}^{\bullet}, m, \mathfrak{d} i^{\bullet}, m\right)$. But $\sigma\left(\mathfrak{Z}^{\bullet}, m, \mathfrak{d} i^{\bullet}, m\right) \subseteq \sigma(\mathfrak{Y}, \overline{\mathfrak{d}})$. Therefore $\mu \in \sigma(\mathfrak{Y}, \overline{\mathfrak{d}})$.

THEOREM 3. Let $(\mathfrak{X}, \mathfrak{d})$ and $(\mathfrak{Y}, \overline{\mathfrak{d}})$ be $\pi$-spectrally connected Banach space complexes parametrized on $\Omega$ and $\Lambda$, respectively, $f: \Omega \rightarrow \Lambda$ a spectral mapping with respect to an $\Omega \times \Lambda$-bicomplex $\left(\mathfrak{Z}, \mathfrak{d}, \mathfrak{d}_{\prime \prime}\right)$ connecting $(\mathfrak{X}, \mathfrak{d})$ and $(\mathfrak{Y}, \overline{\mathfrak{d}})$, and let $\sigma \in \mathfrak{S}^{\pi}$. If $\sigma(\mathfrak{Y}, \overline{\mathfrak{d}})=\Pi_{\Lambda}\left(\sigma\left(\mathfrak{Z}, \mathfrak{d}_{\prime}, \mathfrak{d}_{\prime \prime}^{\prime \prime}\right)\right)$ then

$$
\sigma(\mathfrak{Y}, \overline{\mathfrak{d}}) \subseteq f(\sigma(\mathfrak{X}, \mathfrak{d})),
$$

where $\Pi_{\Lambda}: \Omega \times \Lambda \rightarrow \Lambda$ is the canonical projection.

Proof. Take $\mu \in \sigma(\mathfrak{Y}, \overline{\mathfrak{d}})$. By assumption, $(\lambda, \mu) \in \sigma\left(\mathfrak{Z}, \mathfrak{d} \prime, \mathfrak{d}_{\prime \prime}\right)$ for some $\lambda \in \Omega$. Then $\lambda \in \sigma(\mathfrak{X}, \mathfrak{d})$ by virtue of Proposition 1 . If $f(\lambda) \neq \mu$ then all vertical cohomology complexes of the bicomplex $(\mathfrak{Z}, \mathfrak{d} \prime(\lambda), \mathfrak{d} \prime \prime(\mu))$ are exact by Definition 2. Then $\operatorname{Tot}(\mathfrak{Z}, \mathfrak{d} \prime(\lambda), \mathfrak{d} \prime \prime(\mu))$ is an exact complex by Lemma 4 . But the latter means that $(\lambda, \mu) \notin \sigma_{\mathrm{t}}(\mathfrak{Z}, \mathfrak{d} \prime, \mathfrak{d} \prime \prime)$. In particular, $(\lambda, \mu) \notin \sigma\left(\mathfrak{Z}, \mathfrak{d}_{\prime}, \mathfrak{d}_{\prime \prime}\right)$, a contradiction. Therefore, $\mu=f(\lambda) \in f(\sigma(\mathfrak{X}, \mathfrak{d}))$. Thus $\sigma(\mathfrak{Y}, \overline{\mathfrak{d}}) \subseteq f(\sigma(\mathfrak{X}, \mathfrak{d}))$.

\section{References}

[1] A. A. Dosiev, Ultraspectra of a representation of a Banach Lie algebra, Funct. Anal. and its Appl. 35 (2001), 226-229.

[2] A. A. Dosiev, Algebras of power series in elements of a Lie algebra and Stodkowski spectra, J. Math. Sciences 124 (2004), 4886-4908.

[3] A. A. Dosiev, Spectra of infinite parametrized Banach complexes, J. Operator Theory 48 (3) (2002) 585-614. 
[4] A. A. Dosiev, Fréchet algebra sheaf cohomology and spectral theory, Funct. Anal. and its Appl. (2005) (to appear).

[5] A. A. Dosiev, Cartan-Stodkowski spectra, splitting elements and noncommutative spectral mapping theorems, preprint.

[6] J. Eschmeier and M. Putinar, Spectral Decomposition and Analytic Sheaves, Clarendon Press, Oxford, 1996.

[7] A. S. Fainshtein, Taylor joint spectrum for families of operators generating nilpotent Lie algebra, J. Operator Theory 29 (1993), 3-27.

[8] R. Godement, Algebraic Topology and Sheaf Theory, "IIL", Moscow, 1961 (in Russian).

[9] S. Heinrich, Ultraproducts in Banach space theory, J. Reine Angew. Math. 313 (1980), $72-104$.

[10] A. Ya. Helemskii, The Homology of Banach and Topological Algebras, Kluwer, 1989.

[11] S. S. Kutateladze, Fundamentals of Functional Analysis, Kluwer, 1996.

[12] Z. Słodkowski, An infinite family of joint spectra, Studia Math. 61 (1977), 239-255. 